\title{
MicroRNA399 is involved in multiple nutrient starvation responses in rice
}

\author{
Bin $\mathrm{Hu}^{1}$, Wei Wang ${ }^{1,2}$, Kun Deng ${ }^{3}$, Hua $\mathrm{Li}^{1,2}$, Zhihua Zhang ${ }^{1,2}$, Lianhe Zhang ${ }^{3}$ and \\ Chengcai $\mathrm{Chu}^{1 *}$
}

1 State Key Laboratory of Plant Genomics, National Center for Plant Gene Research (Beijing), Institute of Genetics and Developmental Biology, Chinese Academy of Sciences, Beijing, China, ${ }^{2}$ University of Chinese Academy of Sciences, Beijing, China, ${ }^{3}$ School of Agriculture, Henan University of Science and Technology, Luoyang, China

\section{OPEN ACCESS}

Edited by:

Andrzej Miroslaw Pacak, Adam Mickiewicz University, Poland

Reviewed by:

Janette Palma Fett, Universidade Federal do Rio Grande do Sul, Brazil

Csaba Hornyik,

The James Hutton Institute, UK

*Correspondence: Chengcai Chu,

State Key Laboratory of Plant Genomics, National Center for Plant Gene Research (Beijing), Institute of Genetics and Developmental Biology,

Chinese Academy of Sciences, Beichen West Road No.1, Beijing 100101, China ccchu@genetics.ac.cn

Specialty section:

This article was submitted to Plant Nutrition, a section of the journal Frontiers in Plant Science

Received: 30 December 2014 Accepted: 08 March 2015 Published: 24 March 2015

Citation: Hu B, Wang W, Deng K, Li H, Zhang Z, Zhang L and Chu C (2015) MicroRNA399 is involved in multiple nutrient starvation responses in rice.

Front. Plant Sci. 6:188.

doi: 10.3389/fpls.2015.00188
The increasing evidences have revealed that microRNAs (miRNAs) play significant role in nutrient stress response. Previously, miR399 was documented to be induced by phosphorus $(\mathrm{P})$ starvation and involved in regulating $\mathrm{P}$ starvation responses. To further investigate the function of miR399 in rice (Oryza sativa L.), we performed GeneChip analysis with OsmiR399 over-expressing plants. Interestingly, our results showed that, besides $\mathrm{P}$ starvation responsive genes, the expression of a number of genes involved in iron ( $\mathrm{Fe}$ ), potassium (K), sodium ( $\mathrm{Na}$ ), and calcium (Ca) absorption was dramatically up-regulated in OsmiR399 over-expressing plants. Consistently, the concentrations of $\mathrm{Fe}, \mathrm{K}, \mathrm{Na}$, and $\mathrm{Ca}$ were also increased in OsmiR399 over-expressing plants. The expression of OsmiR399 was also up-regulated by these nutrient starvations, respectively. Moreover, the loss-of-function of LTN1, the down-stream target of OsmiR399, also resulted in the increase of multiple metal elements and the up-regulation of the absorption related genes. These results indicated that OsmiR399 participates in the regulation of multiple nutrient starvation responses, which also gives new view on understanding the interaction among different nutrients mediated by miR399.

Keywords: rice, microRNA399 (miR399), over-expressing plants, gene expression, nutrient starvation response

\section{Introduction}

MicroRNA (miRNA) is a class of 21-24 nucleotides no-coding small RNA, which usually downregulates the expression of target genes at post-transcription level through mRNA degradation or translational repression. More and more evidences have revealed that miRNAs are involved in regulating multiple biological processes, one of which is the stress adaptive response. In plants, the stress-regulated miRNAs have been identified under different stresses, such as drought, cold, salinity, and nutrient deficiency. A great number of miRNAs, for example, miR396, miR168, miR167, miR165, miR319, miR159, miR394, miR156, miR393, miR171, miR158, and miR169 have been identified to be regulated by drought in Arabidopsis (Liu et al., 2008). In rice, 14 miRNAs (miR159, miR169, miR171, miR319, miR395, miR474, miR845, miR851, miR854, miR896, miR901, miR903, miR1026, and miR1125) are significantly induced while 16 miRNAs are significantly repressed by drought (Zhao et al., 2007; Zhou et al., 2010). The expression of miRNAs is also differentially regulated by salt. In Arabidopsis, miR156, miR158, miR159, miR165, miR167, miR168, miR169, miR171, miR319, miR393, miR394, miR396, and miR397 are up-regulated by salt stress while the expression of miR398 is down-regulated (Liu et al., 2008). The expression of miRNAs under cold or heat stress was also studied in 
plants. MiR397, miR169, miR165, miR166, miR393, miR396, and miR408 were shown to be up-regulated by cold stress in Arabidopsis (Sunkar and Zhu, 2004; Liu et al., 2008). The expression of miR156, miR159, miR160, miR166, miR168, miR169, miR393, and miR827 is increased, while miR172 is significantly repressed under heat stress in wheat (Xin et al., 2010).

The availability of nutrients, such as sulfur (S), phosphorus $(\mathrm{P})$, copper $(\mathrm{Cu})$, and nitrogen $(\mathrm{N})$, can affect the expression of miRNAs. $\mathrm{Cu}$ is an important macronutrient for plants and involved in regulating photosynthesis, oxidative responses, and other physiological processes. Among the miRNAs response to nutrient starvation, miR395 and miR399 are two well documented miRNAs involved in $\mathrm{S}$ and $\mathrm{P}$ starvation responses, respectively (Takahashi et al., 2000; Maruyama-Nakashita et al., 2003; Fujii et al., 2005; Chiou et al., 2006). MiR398 is induced under $\mathrm{Cu}$ limiting condition and its up-regulation subsequently increases the $\mathrm{Cu}$ availability (Abdel-Ghany and Pilon, 2008). N starvation induces the expression of miR167a and represses the expression of its target, $A R F 8$, which regulates the lateral root initiation (Gifford et al., 2008). In contrast, miR169 is significantly repressed by $\mathrm{N}$ starvation while NFYA, the target of miR169, is induced (Zhao et al., 2011).

$\mathrm{S}$ is a constituent of two amino acids, cysteine and methionine, and therefore, it is an important component of protein. In plants, $\mathrm{S}$ is acquired and assimilated mainly in the form of sulfate, which is mediated by sulfate transporters and ATP sulphurylase (APS), respectively. MiR395 is involved in regulating $S$ assimilation by targeting the sulfate transporter gene SULTR2;1 and APS genes (Takahashi et al., 2000; Maruyama-Nakashita et al., 2003). The expression of miR395 is induced by long-term sulfate deficiency while expression of SULTR2;1 is repressed. The down-regulation of SULTR2; 1 by sulfate deficiency is only detected in the shoots whereas its mRNA is greatly accumulated in the roots, suggesting that the degradation of SULTR2;1 by miR395 mainly occurs in the shoots (Takahashi et al., 1997, 2000). In addition to SULTR2;1, miR395 is also predicted to target several APS genes (APS1, APS2, APS3, and APS4), two (APS1 and APS4) of which were validated by 5'-RACE (Jones-Rhoades and Bartel, 2004; Allen et al., 2005). APS1, APS3, and APS4 were predicted to be localized at the plastid, while APS2 localized at the cytoplasm (Hatzfeld et al., 2000). Most of the sulfate is reduced in the plastid by APS, which indicates that miR395 is an important regulator of sulfate assimilation in plastids (Rotte and Leustek, 2000).

$\mathrm{P}$ is one of the most significant macronutrients for plants, which constitutes many important biological molecules such as nucleotides, phospholipids, and ATP. Inorganic phosphate (Pi) is the major form of available $\mathrm{P}$ for plants in the soil. However, in most natural soils, the concentration of Pi is very low and consequently results in the $\mathrm{P}$ starvation condition. Accordingly, plants have also evolved P starvation responses to increase Pi availability under this condition (Raghothama, 1999). MiR399 is an important component involved in the $\mathrm{P}$ starvation signaling. It consists of 6 (miR399a-f) and 11 (OsmiR399a-k) members in Arabidopsis and rice, respectively. The function of miR399 in $\mathrm{P}$ starvation signaling was firstly studied in Arabidopsis. Under P starvation condition, the expression of miR399 is dramatically induced while the expression of its target gene $\mathrm{PHO} 2$ (UBC24) is decreased (Fujii et al., 2005; Chiou et al., 2006). Both miR399 over-expressing plants and pho2 mutant showed Pi overaccumulation in the shoots (Fujii et al., 2005; Chiou et al., 2006). PHO2 encodes an ubiquitin-conjugating enzyme (E2), which is involved in ubiquitin-mediated protein degradation. Recent work showed that the degradation of PHO1 and PT2, two important Pi transporters controlling Pi homeostasis, is dependent on $\mathrm{PHO} 2$, and the ubiquitin ligase (E3) NLA is proved to coordinate with $\mathrm{PHO} 2$ to mediate this process (Liu et al., 2012; Lin et al., 2013; Park et al., 2014). In addition, the expression of miR399 is up-regulated by the Myb transcription factor PHR1, which is

TABLE 1 | The up-regulated genes involved in nutrient absorption in OsmiR399-ox plants (f8) from GeneChip assay.

\begin{tabular}{|c|c|c|c|}
\hline Gene name & Loc. No. & Fold change & $P$-value \\
\hline \multicolumn{4}{|l|}{ P ABSORPTION RELATED GENES } \\
\hline Putative high-affinity phosphate transporter & LOC_Os08g45000 & 3.9 & 0.00002 \\
\hline Putative inorganic phosphate transporter & LOC_Os10g30770 & 2.6 & 0.00002 \\
\hline Phosphate transporter & LOC_Os03g05640 & 2.3 & 0.00002 \\
\hline Putative phosphate transporter & LOC_Os10g30790 & 1.5 & 0.00004 \\
\hline Partial mRNA for phospholipase C & LOC_Os12g37560 & 1.3 & 0.001651 \\
\hline Secretory acid phosphatase precursor & LOC_Os12g44020 & 1.2 & 0.00003 \\
\hline Secretory acid phosphatase precursor & LOC_Os12g44020 & 1.2 & 0.00003 \\
\hline \multicolumn{4}{|c|}{ METAL ELEMENTS ABSORPTION RELATED GENES } \\
\hline Nicotianamine synthase (OsNAS1) & LOC_Os03g19420 & 2.1 & 0.000033 \\
\hline Calcium-transporting ATPase (CaC) & LOC_Os01g71240 & 3.1 & 0.00002 \\
\hline
\end{tabular}

The P-value was determined by One-Way ANOVA. The fold change represents the increased folds of gene expression in OsmiR399-ox plants compared with wild type. 
the key transcription factor activating the expression of $\mathrm{P}$ starvation responsive genes (Rubio et al., 2001; Nilsson et al., 2007). In rice, the $\mathrm{P}$ starvation signal transduction pathway is conserved with that in Arabidopsis, which mainly consists of OsPHR2, OsmiR399, OsPHO2 (LTN1), and the downstream P starvation responsive genes (Zhou et al., 2008; Hu et al., 2011).

Our previous work revealed that OsmiR399 over-expression resulted in the increase of $\mathrm{Pi}$ accumulation in rice (Hu et al., 2011). Here we further showed that the concentration of several other nutrients including iron $(\mathrm{Fe})$, potassium $(\mathrm{K})$, sodium $(\mathrm{Na})$, and calcium $(\mathrm{Ca})$ were also increased in OsmiR399 over-expressing plants and OsmiR399s were coordinately up-regulated by these nutrient starvations, respectively, indicating that miR399 expression responds to multiple nutrient starvations and is involved in the regulation of multi-nutrient absorption.

\section{Materials and Methods}

\section{Plant Materials and Growth Conditions}

OsmiR399 over-expressing (ox) plants used in this study including OsmiR399f-ox plants (f1 and f8) and OsmiR399j-ox plants (j6 and j8) were generated in Nipponbare background as previously described (Hu et al., 2011). The ltnl mutant was identified from our rice mutant population (Ma et al., 2009) by screening the mutants with leaf senescence phenotype. The ln 1 mutant was shown to over-accumulate $\mathrm{Pi}$ in the leaf, which resulted in the leaf tip necrosis phenotype ( $\mathrm{Hu}$ et al., 2011).

OsmiR399-ox plants, $\ln 1$ mutant, and wild type Nipponbare were grown in the $1 / 2$ strength Hoagland solution with minor modification, the full nutrition solution contains $2.5 \mathrm{mM}$ $\mathrm{KNO}_{3}, 1 \mathrm{mM} \mathrm{Ca}\left(\mathrm{NO}_{3}\right)_{2}, 1 \mathrm{mM} \mathrm{FeSO} 4$-EDTA, $1 \mathrm{mM} \mathrm{MgSO} 4$, $0.5 \mathrm{mM} \mathrm{NH} \mathrm{H}_{2} \mathrm{PO}_{4}, 20 \mathrm{mM} \mathrm{H}_{3} \mathrm{BO}_{4}, 5 \mu \mathrm{M} \mathrm{MnCl}_{2}, 0.38 \mu \mathrm{M}$ $\mathrm{ZnSO}_{4}, 0.16 \mu \mathrm{M} \mathrm{CuSO}_{4}, 0.06 \mu \mathrm{M} \mathrm{Na}_{2} \mathrm{MoO}_{4}$. The nutrient starvation solutions were designed by using $1 / 2$ strength modified Hoagland solution lacking specific elements ( $\mathrm{P}, \mathrm{Fe}, \mathrm{Na}$ or $\mathrm{Ca}$ ). The hydroponic culture was adjusted to $\mathrm{pH} 6.0$ with $2 \mathrm{M} \mathrm{NaOH}$ before use and the medium was changed every 3 days. The hydroponic cultured seedlings were grown in an incubator with a $12 \mathrm{~h}$ day $\left(30^{\circ} \mathrm{C}\right) / 12 \mathrm{~h}$ night $\left(28^{\circ} \mathrm{C}\right)$ photoperiod, approximately $200 \mu \mathrm{mol}$ photons $\mathrm{m}^{-2} \mathrm{~s}^{-1}$ light intensity, and approximately $70 \%$ humidity. The seedlings were maintained under the treatments for 2-week (after germination to sample harvesting) and the samples under different treatments were collected at the same moment.

\section{RNA Isolation, cDNA Preparation, and Quantitative RT-PCR}

Total RNA was extracted using the TRIzol reagent (Invitrogen). Approximately $2 \mu \mathrm{g}$ of the total RNA treated with DNase I was used to synthesize the first-strand cDNA using oligo $(\mathrm{dT})_{18}$ as primer. The product of first-strand CDNA was used as the template for the PCR. For qRT-PCR, SYBR Green I was added to the reaction mix and run on a Chromo4 real-time PCR detection system (Bio-Rad, CFX96) according to the manufacturer's instructions. The data were analyzed with Opticon monitor software (Bio-Rad). The expression of miRNAs was investigated by analyzing the expression of their precursors (pri-miRNA). The PCR products of each pri-miRNA were sequenced to confirm the specific amplification of the corresponding pri-miRNA. For the mature miRNA assay in the ox lines, the miRNA First-strand cDNA Synthesis kit (Tiangen, KR201) and the miRNA qPCR Detection kit (Tiangen, KR201) were used according to the manufacturer's instructions. Briefly, the miRNAs are polyadenylated by poly(A) polymerase and subsequently converted into cDNA by reverse transcriptase with oligodT priming. The miRNAs are converted into cDNA by reverse transcriptase using both oligo$\mathrm{dT}$ and random priming. The cDNA is then used for real-time PCR quantification of mature miRNA with the forward miRNA specific primer (with the conserved mature OsmiR399 sequence) and the reverse universal primer. Rice ACTIN1 was used as the internal control in all analyses. The primers for qRT-PCR are listed in Supplementary Table 1. Each gene expression assay with qRT-PCR was replicated for 3 times.

\section{Affymetrix ATH1 GeneChip Assay}

The roots of OsmiR399-ox plants (f8) and Nipponbare cultured in $1 / 2$ Hoagland solution for 2 -week were collected for

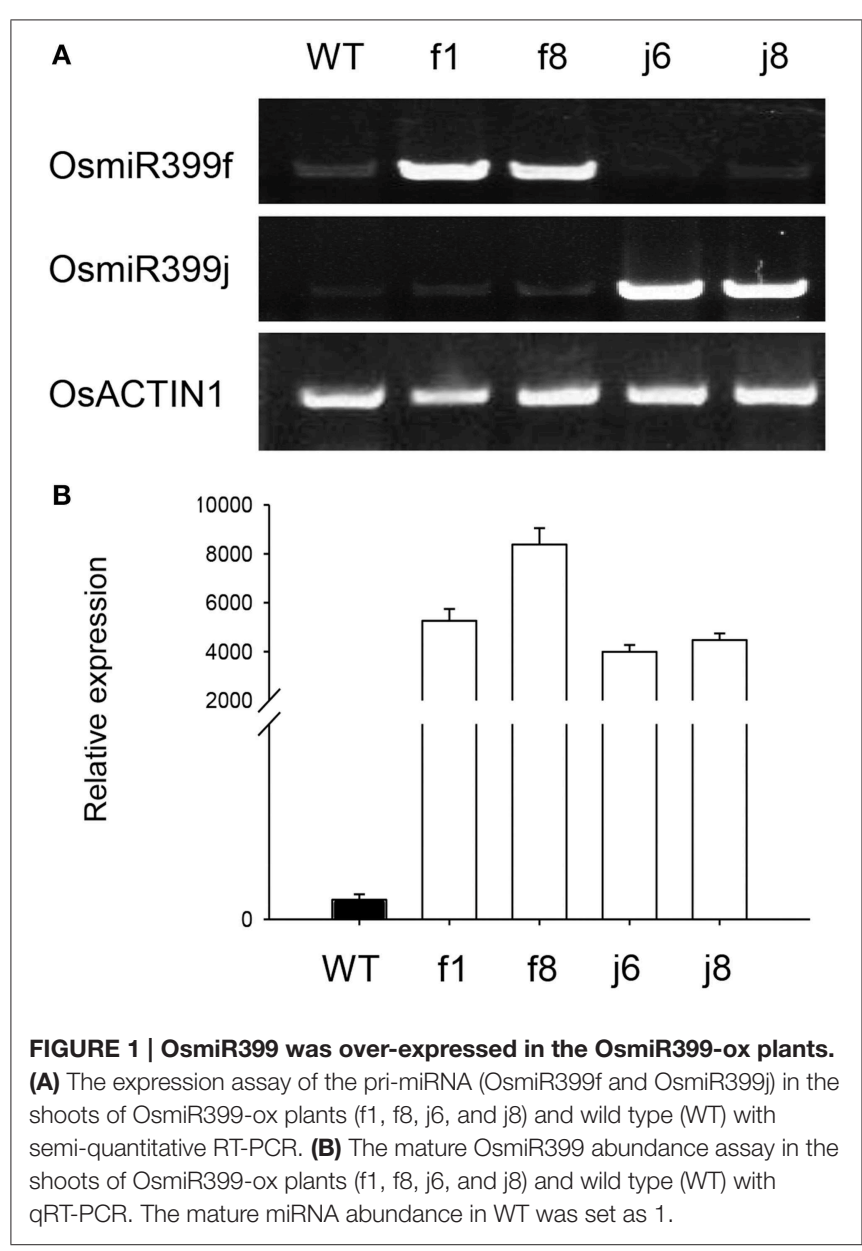




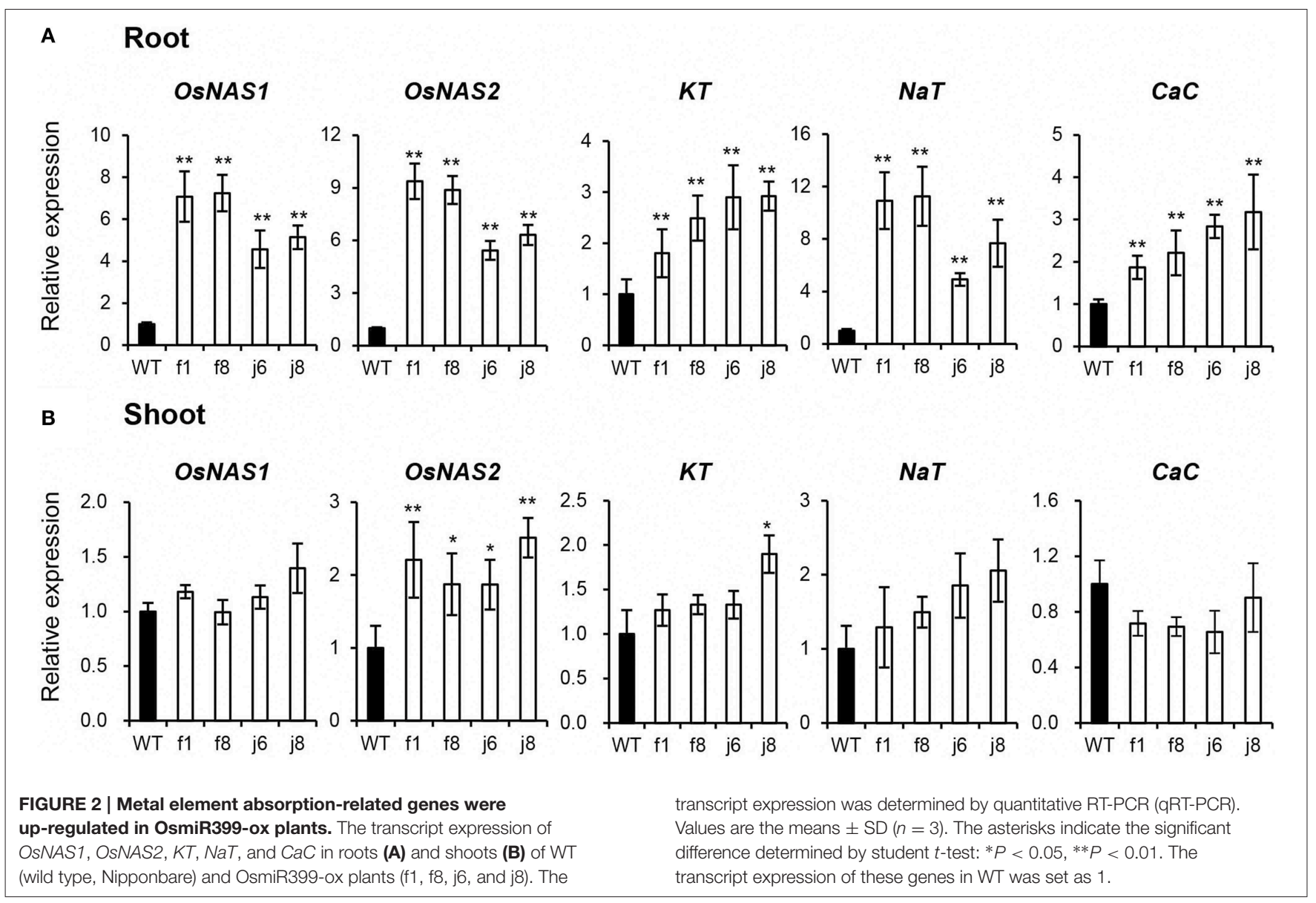

GeneChip analysis. The total RNA was extracted with the TRIzol reagent (Invitrogen) according to the manufacturer's instructions. Affymetrix array service was provided by Capitalbio Corporation, Beijing. The GeneChip data was listed in Supplementary Table 2.

\section{Metal Element Concentration Determination}

Roots and shoots of 2-week-old seedlings grown in $1 / 2$ strength Hoagland solution were dried at $70^{\circ} \mathrm{C}$ for $48 \mathrm{~h}$ in an oven. Exactly $80 \mathrm{mg}$ samples were digested with $13 \mathrm{ml}$ concentrated $\mathrm{HNO}_{3}$ and $2 \mathrm{ml} 30 \% \mathrm{H}_{2} \mathrm{O}_{2}$ at $140^{\circ} \mathrm{C}$ for $30 \mathrm{~min}$. Digested solution volumes were adjusted to $25 \mathrm{ml}$ with de-ionized water. The metal elements were determined with ICP-OES (PE, Optima 2000 DV).

\section{Results}

\section{OsmiR399 Participates in Regulating Multiple Nutrient Absorption Genes}

To investigate how OsmiR399 regulates the expression of downstream genes, we performed GeneChip analysis using OsmiR399ox plants (f8) and its corresponding wild type Nipponbare. The materials of roots were collected and assayed with Affymetrix GeneChip. As expected, the expression of Pi absorption-related genes was significantly increased in the roots of OsmiR399-ox plants (Table 1). Interestingly, several genes probably involved in metal element absorption were also largely up-regulated in OsmiR399-ox plants (Table 1), including the genes encoding nicotianamine synthase (OsNAS1 and OsNAS2), potassium transporter $(\mathrm{KT})$, sodium transporter $(\mathrm{NaT})$, and calcium channel $(\mathrm{CaC})$, which gives the hint for the connection between miR399 and multiple nutrient absorption in rice.

To confirm the result obtained from GeneChip, four independent OsmiR399-ox lines including f1, f8, j6, and j8 were further analyzed. Both the pri-miRNA and mature miRNA were significantly over-expressed in theses ox lines (Figure 1). The expression assay of the metal absorption related genes in these ox lines showed that OsNAS1, OsNAS2, KT, NaT, and $\mathrm{CaC}$ were significantly increased in the roots of OsmiR399-ox lines (Figure 2A), whereas in shoots, except OsNAS2, no significant alteration was observed in the expression of these genes in OsmiR399-ox lines (Figure 2B). These data indicates that regulation of these genes by OsmiR399 occurs mainly in the roots.

\section{$\mathrm{Fe}, \mathrm{K}, \mathrm{Na}$, and $\mathrm{Ca}$ Concentrations were Increased in OsmiR399-ox Plants}

The up-regulation of the related genes suggested that the absorption of multiple nutrients is increased by OsmiR399 overexpression. Thus, the concentrations of multiple elements were 
further determined in OsmiR399-ox plants (f1, f8, j6, and j8) and the wild-type Nipponbare. The P concentration was largely increased in the shoots of all OsmiR399-ox plants while no significant alteration was found in the roots (Figure 3). In contrast, the Fe concentration was only increased in the roots of OsmiR399-ox plants (Figure 3A). The K concentration was also specifically increased in the shoots, and $\mathrm{Na}$ and $\mathrm{Ca}$ concentrations were increased in both roots and shoots of OsmiR399-ox plants (Figure 3). These results provided further evidence that OsmiR399 is involved in regulating the absorption of multiple nutrients, and also suggested that the mechanisms of regulation by OsmiR399 differ among different nutrients.

\section{OsmiR399 can be Induced by $\mathrm{Fe}, \mathrm{K}, \mathrm{Na}$, or $\mathrm{Ca}$ Starvation}

As over-expression of OsmiR399 resulted in increased absorption of multiple nutrients, we further investigated whether OsmiR399 is responsive to these nutrient starvations. Pri-miRNA is the primary transcript of the mature miRNA. The mature miRNAs have very high sequence similarity between different members while the sequence similarity of the pri-miRNAs is relatively low except in the mature miRNA region. This sequence heterology can help to detect the expression of different members of miR399 family via analyzing the expression of the corresponding primiRNAs. Thereby, the expression of seven OsmiR399 members (OsmiR399a, OsmiR399d, OsmiR399e, OsmiR399f, OsmiR399i, OsmiR399j, and OsmiR399k) was examined by analyzing the expression of their corresponding pri-miRNAs with qRT-PCR. Consistent with the previous result, most members of OsmiR399 can be induced by $\mathrm{P}$ starvation in the shoots, except three members (OsmiR399d, OsmiR399f, and OsmiR399j) which were significantly induced in the roots (Figure 4A). To our surprise, most OsmiR399s also can be significantly induced by Fe starvation in both roots and shoots and the induced level is much higher in shoots than in roots (Figure 4B). Notably, the induction of OsmiR399 by Fe starvation is almost comparable to that by $\mathrm{P}$ starvation in the shoots, indicating OsmiR399 is an important component involved in Fe starvation responses. In shoots, most members of OsmiR399 were induced by K starvation, especially OsmiR399a and OsmiR399k, whereas in roots, only OsmiR399d was up-regulated by K starvation (Figure 5A). Under Na starvation, the up-regulation of OsmiR399 was also observed in both

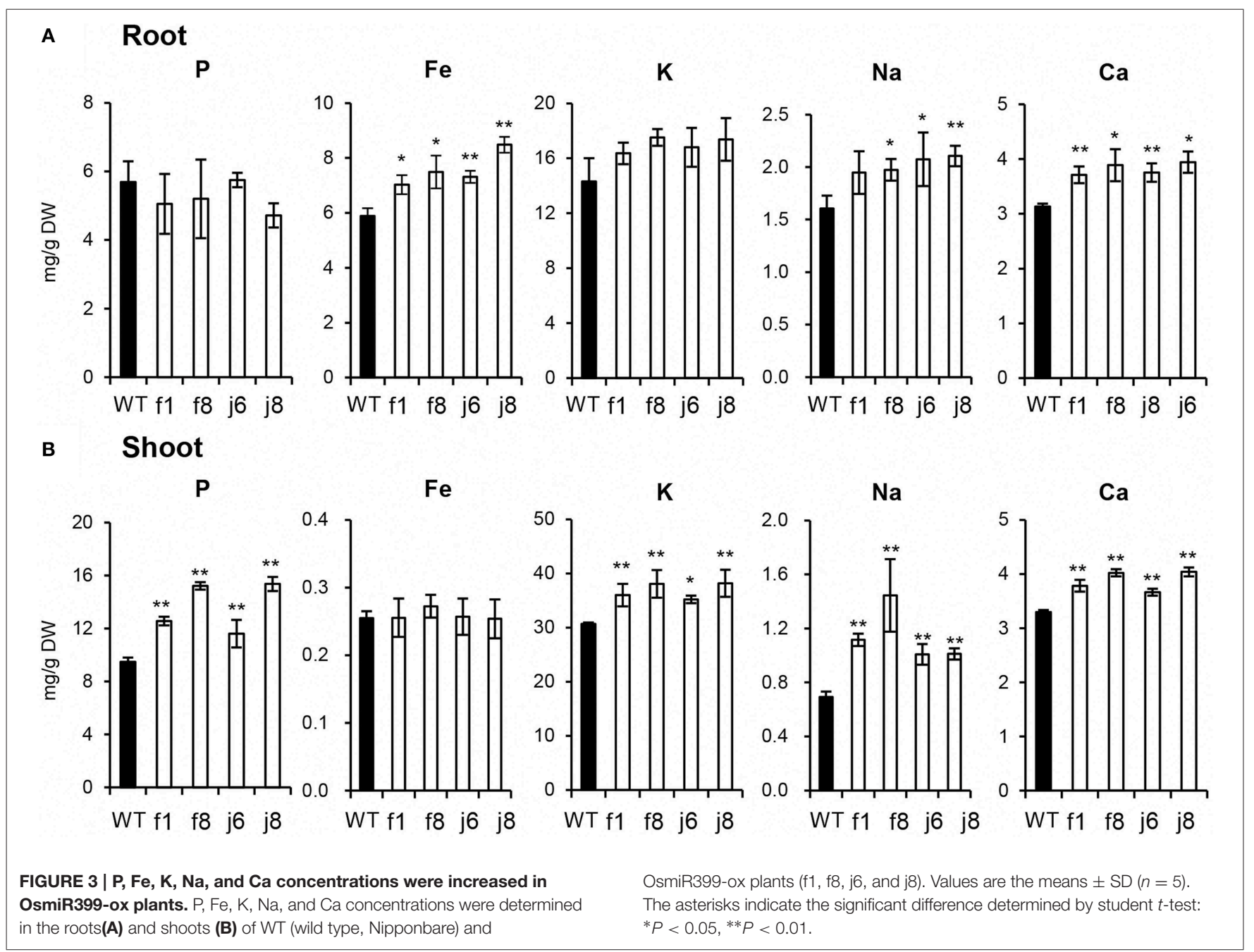




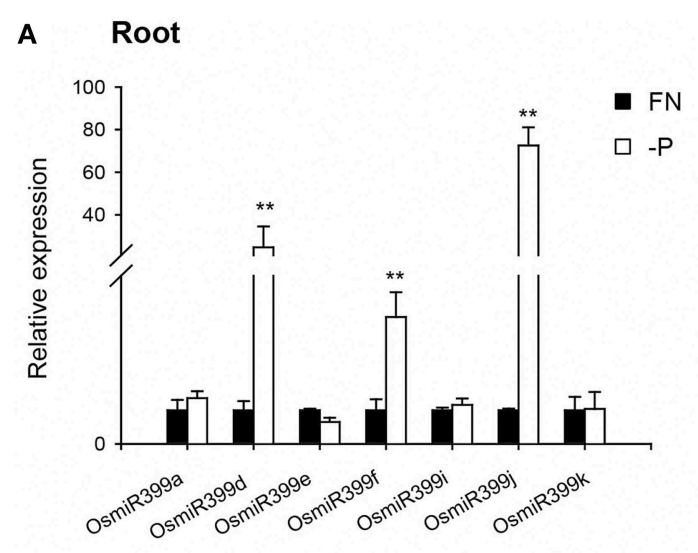

\section{Shoot}

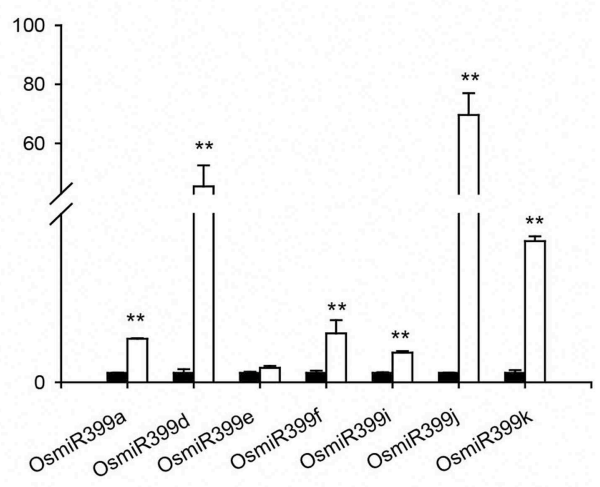

B Root

Shoot
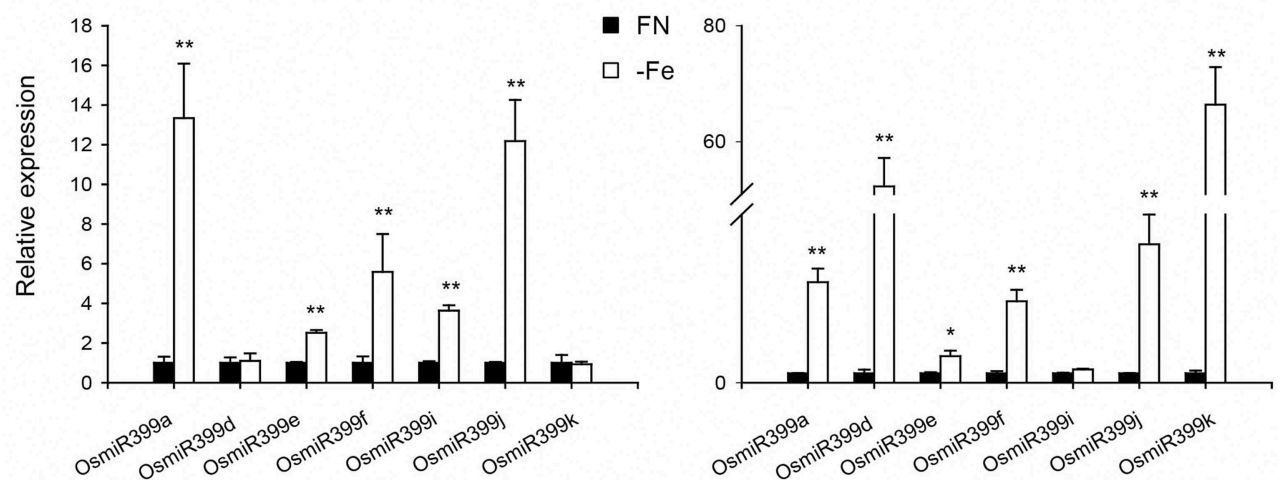

FIGURE 4 | OsmiR399s were induced by $P$ or Fe starvation. (A) The transcript expression assay of OsmiR399 under $P$ starvation (-P). (B) The transcript expression assay of OsmiR399 under Fe starvation (-Fe). The expression of 7 OsmiR399 members (OsmiR399a, OsmiR399d, OsmiR399e, OsmiR399f, OsmiR399i, OsmiR399j, OsmiR399k) were examined by

shoots and roots. However, only two specific OsmiR399 transcripts were induced by Ca starvation in roots (OsmiR399j and OsmiR399k) and shoots (OsmiR399d and OsmiR399j), respectively (Figures 5B,C). It is noted that the induction of OsmiR399 under $\mathrm{K}, \mathrm{Na}$, or $\mathrm{Ca}$ starvation was much less than that under $\mathrm{P}$ starvation. The above results demonstrated that OsmiR399 is an important component involved in multiple nutrient starvation responses.

\section{LTN1 is Involved in Regulation of Multiple Nutrients Absorption}

As LTN1 is the downstream target of OsmiR399 (Hu et al., 2011), it is possible that LTN1 is also involved in the regulation of multiple nutrient absorption. To verify this speculation, the nutrient concentrations were determined and the expression of the related genes was examined in the $\ln 1$ mutant. The results showed that the Fe concentration was increased in the roots of ltn1 mutant (Figure 6A), which is consistent with the result from OsmiR399-ox plants. The concentration of $\mathrm{K}$ was only increased in the shoots, while $\mathrm{Na}$ and $\mathrm{Ca}$ concentrations were increased in both roots and shoots of $l n n 1$ mutant (Figure 6A). Consistently, the transcript expression of OsNAS1, OsNAS2, KT, NaT, and $\mathrm{CaC}$ was also significantly increased in the roots of $l n n 1$ mutant (Figure 6B). These results indicated that LTN1 is involved in the regulation of multiple nutrient absorptions.

\section{Discussion}

Previous work showed that miR399 is a significant component mediating $\mathrm{P}$ starvation responses. In this work, our data further demonstrated that miR399 is also involved in regulating multiple nutrient starvation responses. The OsmiR399-ox plants displayed increased expression of genes involved in metal element absorption. OsNAS1 and OsNAS2, the nicotianamine synthase genes, were up-regulated in OsmiR399-ox plants. Previous work suggested that OsNAS1 and OsNAS2 mediate the longdistance transport of Fe (Inoue et al., 2003), while our results indicated that their up-regulation may also lead to increased $\mathrm{Fe}$ accumulation in roots. The expression of the $\mathrm{K}$ and $\mathrm{Na}$ transporter genes, $K T$ and $\mathrm{NaT}$, was also elevated in OsmiR399-ox 

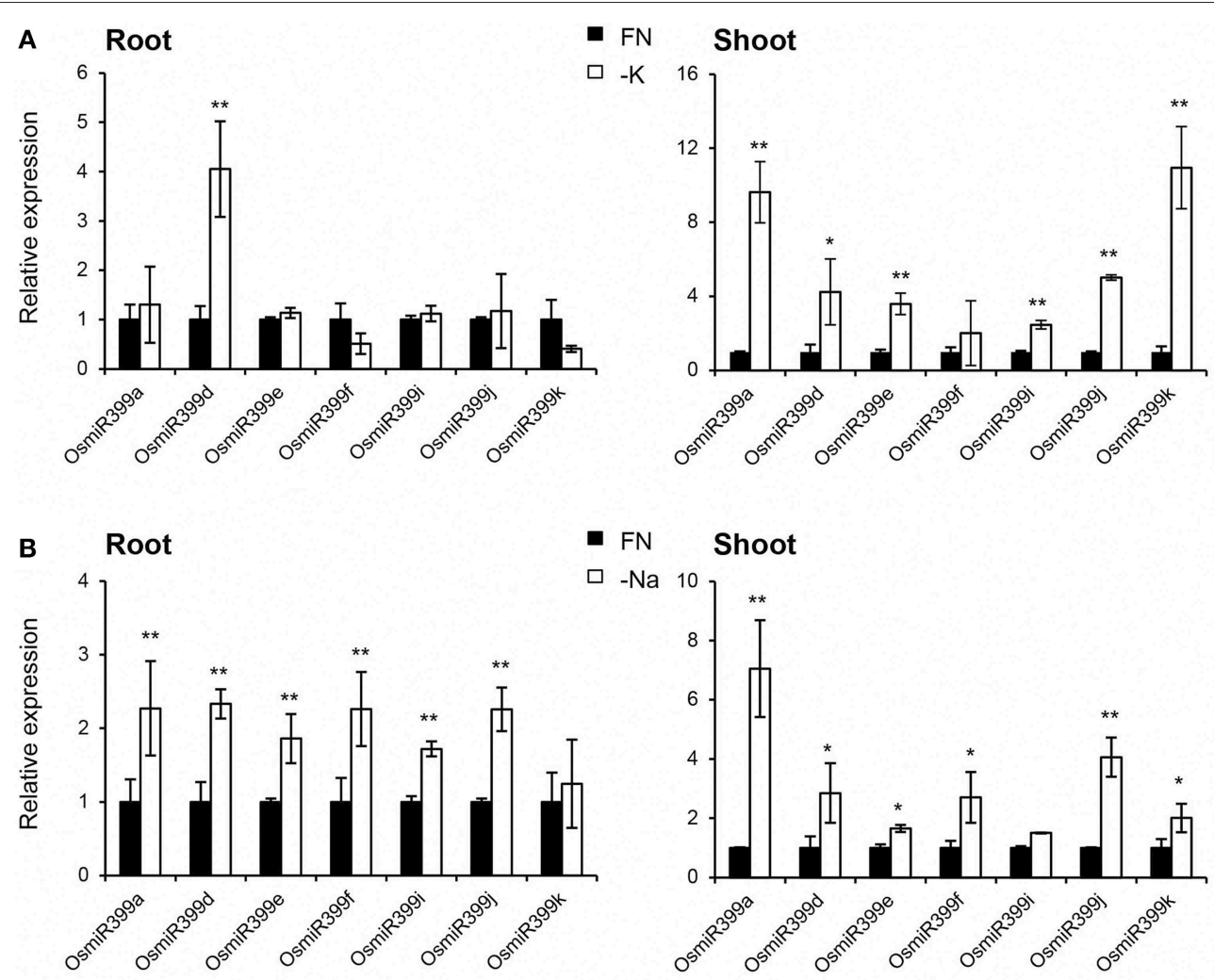

- FN Shoot

$\square-\mathrm{Na} 10$
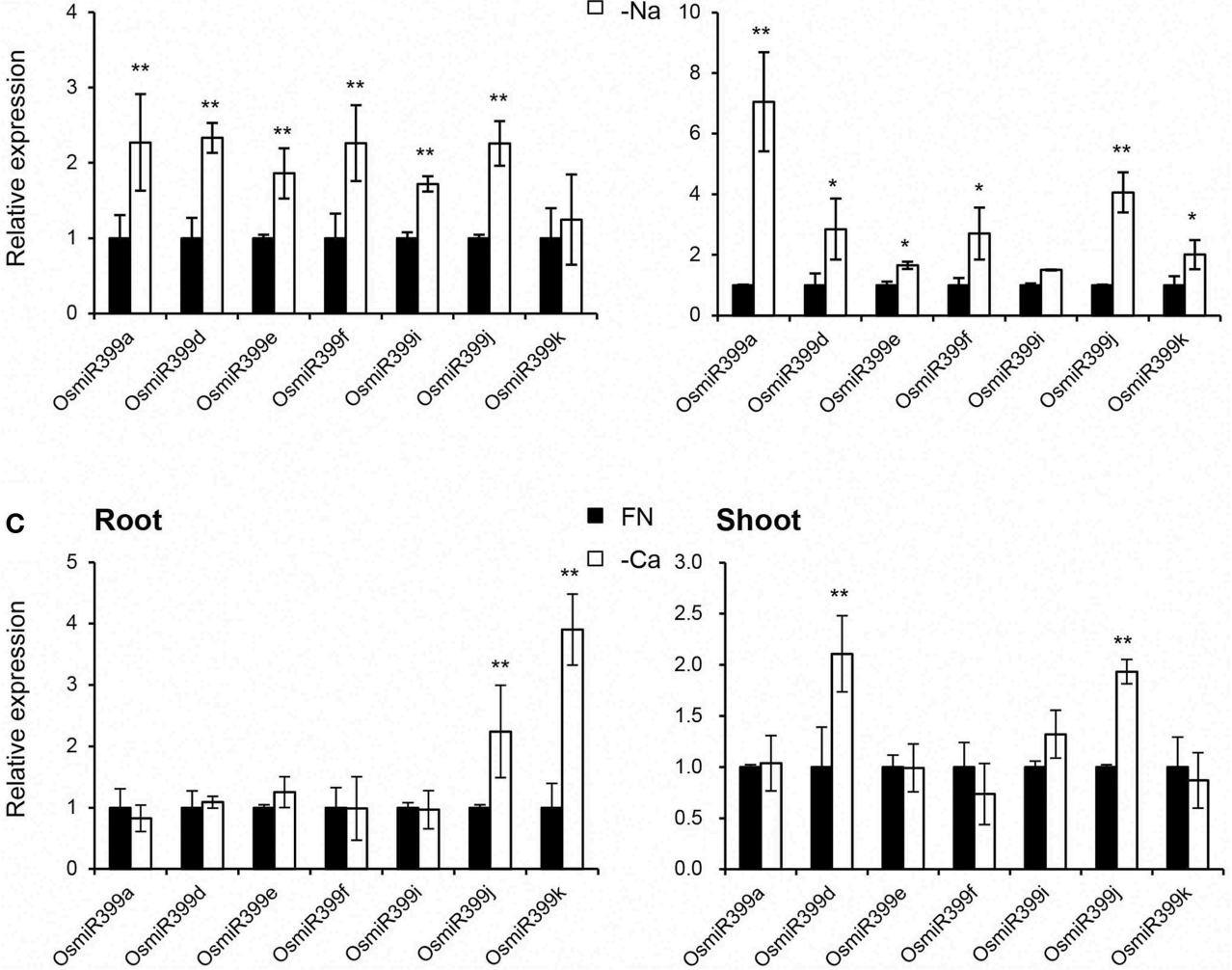

- FN Shoot

FIGURE 5 | OsmiR399s were induced by $\mathrm{K}$, $\mathrm{Na}$ or $\mathrm{Ca}$ starvation.

(A) The transcript expression assay of OsmiR399 under $\mathrm{K}$ starvation (-K). (B) The transcript expression assay of OsmiR399 under $\mathrm{Na}$ starvation (-Na). (C) The transcript expression assay of OsmiR399 under Ca starvation (-Ca). The expression of seven OsmiR399 members (OsmiR399a, OsmiR399d, OsmiR399e, OsmiR399f, OsmiR399i,
OsmiR399j, OsmiR399k) were examined by analyzing the expression of their precursors (pri-miRNA). FN, full nutrition condition. The transcript expression was determined by qRT-PCR. Values are the means $\pm S D$ $(n=3)$. The asterisks indicate the significant difference determined by student $t$-test: ${ }^{*} P<0.05,{ }^{*} P<0.01$. The transcript expression of OsmiR399 under FN was set as 1. plants, resulting in the increase of $\mathrm{K}$ and $\mathrm{Na}$ concentrations. The increase of $\mathrm{Ca}$ concentration in OsmiR399-ox plants was possibly associated with the enhanced expression of the calcium channel encoding gene $\mathrm{CaC}$. In Arabidopsis, it was reported that miR399 is specifically induced by $\mathrm{P}$ starvation, but not by $\mathrm{N}, \mathrm{S}$, or K starvation (Fujii et al., 2005). Our results revealed that, besides $\mathrm{P}$ starvation, the expression of OsmiR399 is also responsive to $\mathrm{Fe}, \mathrm{K}, \mathrm{Na}$, or $\mathrm{Ca}$ starvation. However, except $\mathrm{Fe}$ starvation, the induction of OsmiR399 by other metal element starvations was significantly lower than that by $\mathrm{P}$ starvation. Since there is a strong interaction between different nutrients, it is possible that the deficiencies of these metal elements result in the repression of $\mathrm{Pi}$ uptake. Whether OsmiR399 is directly induced by these metal element starvations or by $\mathrm{P}$ starvation caused by these nutrient deficiencies needs to be further investigated. 


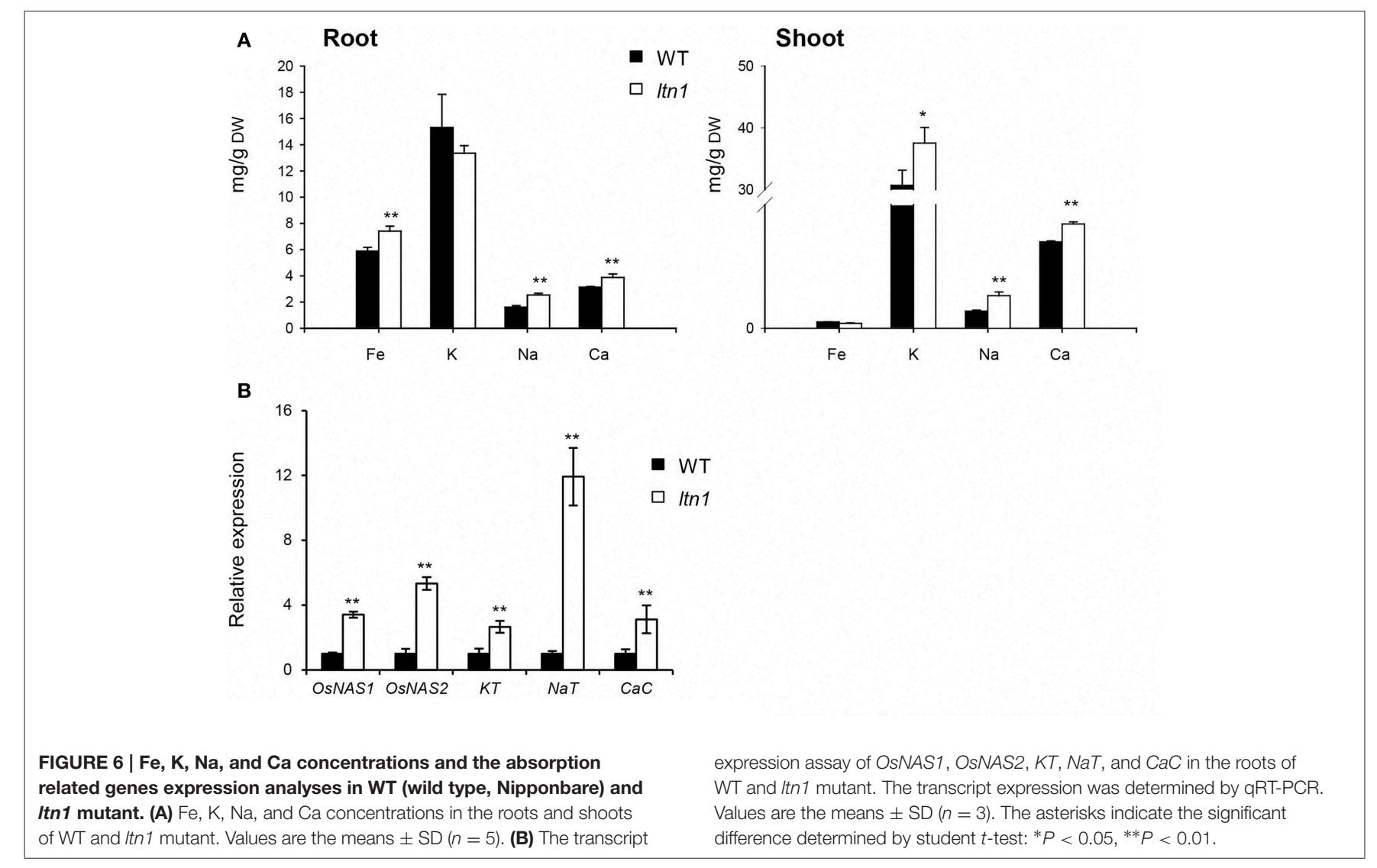

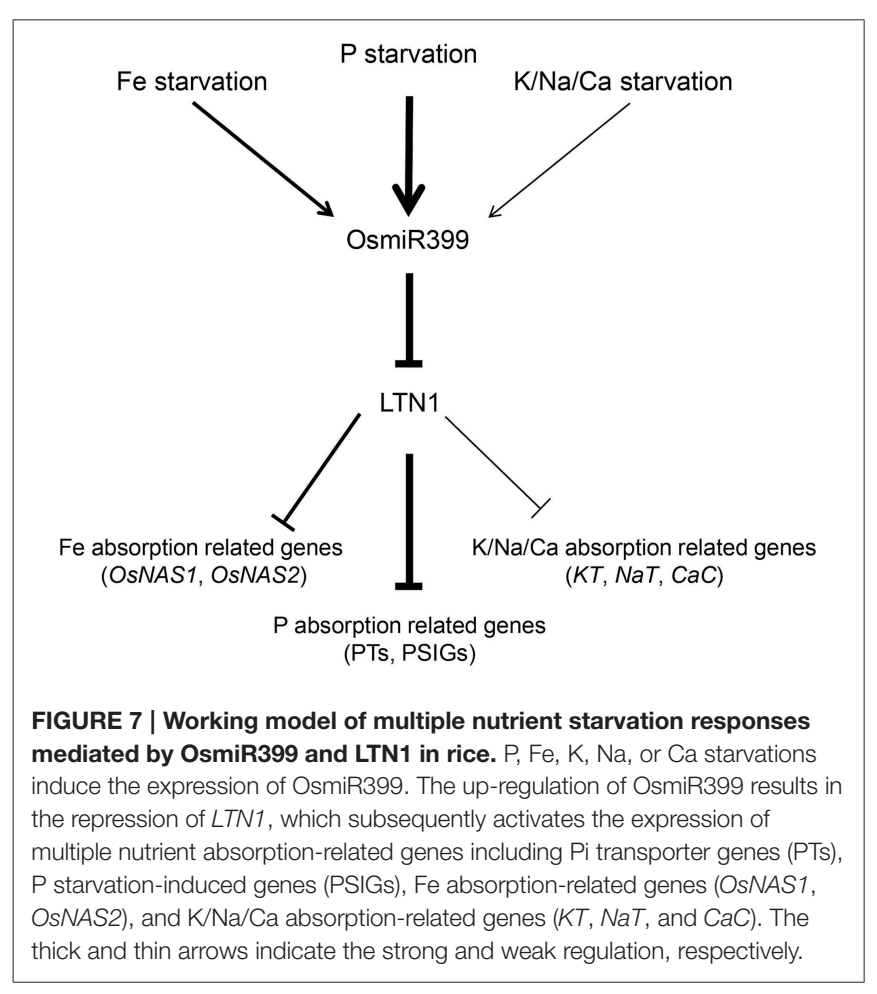

Since miRNAs usually function as the negative regulators (Fujii et al., 2005; Chiou et al., 2006; Hu et al., 2011), it is reasonable to speculate that these up-regulated genes in OsmiR399-ox plants are probably under the control of LTN1. Our previous work also showed that, in the roots of ltn 1 mutant, Fe concentration was increased while the genes involved in $\mathrm{Fe}$ assimilation including OsNAS1 and OsNAS2 were up-regulated (Hu et al., 2011), further suggesting that OsmiR399 regulates metal element absorption through repressing the expression of LTN1. In this work, we showed that LTN1 also regulates $\mathrm{K}, \mathrm{Na}$, and $\mathrm{Ca}$ absorption. These results indicated that OsmiR399 and LTN1 possibly play a pivotal role in regulating multiple nutrient responses in plants. Based on these results, we proposed the following model (Figure 7): $\mathrm{P}, \mathrm{Fe}, \mathrm{K}, \mathrm{Na}$, or Ca starvation induces the expression of OsmiR399, the up-regulation of OsmiR399 results in the repression of $L T N 1$, which subsequently activates the expression of multiple nutrient absorption-related genes including $\mathrm{Pi}$ transporter genes (PTs), P starvation-induced genes (PSIGs), $\mathrm{Fe}$ absorption-related genes (OsNAS1, OsNAS2), and $\mathrm{K} / \mathrm{Na} / \mathrm{Ca}$ absorption-related genes ( $\mathrm{KT}, \mathrm{NaT}$, and $\mathrm{CaC})$. The up-regulation of these genes will enhance the absorption of multiple nutrients, which finally improves the adaptive ability of plants under nutrient starvation condition. Although P starvation can directly induce the expression of miR399, it is unknown whether induction of miR399 by other nutrient starvation is direct or indirect. 
As OsmiR399 is the target of OsPHR2 (Zhou et al., 2008), it is possible that OsPHR2 is involved in this process. Investigation of the expression of these nutrient absorption related genes and the concentrations of multiple nutrients in OsPHR2ox/RNAi transgenic plants will help to complete the signaling pathway of nutrient starvation responses mediated by miR399.

\section{Conclusion}

The miR399 was previously demonstrated to mediate the P starvation signal transduction in both Arabidopsis and rice. Our work further showed that miR399 is also involved in regulating absorption of multiple nutrients and its expression is regulated by the status of these nutrients in rice. This result extends our knowledge on the function of miR399 in plant nutrition and also

\section{References}

Abdel-Ghany, S. E., and Pilon, M. (2008). MicroRNA-mediated systemic downregulation of copper protein expression in response to low copper availability in Arabidopsis. J. Biol. Chem. 283, 15932-15945. doi: 10.1074/jbc.M801406200

Allen, E., Xie, Z. X., Gustafson, A. M., and Carrington, J. C. (2005). microRNAdirected phasing during trans-acting siRNA biogenesis in plants. Cell 121, 207-221. doi: 10.1016/j.cell.2005.04.004

Chiou, T. J., Aung, K., Lin, S. I., Wu, C. C., Chiang, S. F., and Su, C. L. (2006). Regulation of phosphate homeostasis by microRNA in Arabidopsis. Plant Cell 18, 412-421. doi: 10.1105/tpc.105.038943

Fujii, H., Chiou, T. J., Lin, S. I., Aung, K., and Zhu, J. K. (2005). A miRNA involved in phosphate-starvation response in Arabidopsis. Curr. Biol. 15, 2038-2043. doi: 10.1016/j.cub.2005.10.016

Gifford, M. L., Dean, A., Gutierrez, R. A., Coruzzi, G. M., and Birnbaum, K. D. (2008). Cell-specific nitrogen responses mediate developmental plasticity. Proc. Natl. Acad. Sci. U.S.A. 105, 803-808. doi: 10.1073/pnas.0709559105

Hatzfeld, Y., Lee, S., Lee, M., Leustek, T., and Saito, K. (2000). Functional characterization of a gene encoding a fourth ATP sulfurylase isoform from Arabidopsis thaliana. Gene 248, 51-58. doi: 10.1016/S0378-1119(00)00132-3

Hu, B., Zhu, C. G., Li, F., Tang, J. Y., Wang, Y. Q., Lin, A. H., et al. (2011). LEAF TIP NECROSIS1 plays a pivotal role in the regulation of multiple phosphate dtarvation responses in rice. Plant Physiol. 156, 1101-1115. doi: 10.1104/pp.110.170209

Inoue, H., Higuchi, K., Takahashi, M., Nakanishi, H., Mori, S., and Nishizawa, N. K. (2003). Three rice nicotianamine synthase genes, OsNAS1, OsNAS2, and OsNAS3 are expressed in cells involved in long-distance transport of iron and differentially regulated by iron. Plant J. 36, 366-381. doi: 10.1046/j.1365313X.2003.01878.x

Jones-Rhoades, M. W., and Bartel, D. P. (2004). Computational identification of plant MicroRNAs and their targets, including a stress-induced miRNA. Mol. Cell 14, 787-799. doi: 10.1016/j.molcel.2004.05.027

Lin, W. Y., Huang, T. K., and Chiou, T. J. (2013). NITROGEN LIMITATION ADAPTATION, a target of microRNA827, mediates degradation of plasma membrane-localized phosphate transporters to maintain phosphate homeostasis in Arabidopsis. Plant Cell 25, 4061-4074. doi: 10.1105/tpc.113.116012

Liu, T. Y., Huang, T. K., Tseng, C. Y., Lai, Y. S., Lin, S. I., Lin, W. Y., et al. (2012). PHO2-dependent degradation of PHO1 modulates phosphate homeostasis in Arabidopsis. Plant Cell 24, 2168-2183. doi: 10.1105/tpc.112.096636

Liu, H. H., Tian, X., Li, Y. J., Wu, C. A., and Zheng, C. C. (2008). Microarraybased analysis of stress-regulated microRNAs in Arabidopsis thaliana. RNA 14, 836-843. doi: 10.1261/rna.895308

Ma, Y. M., Liu, L., Zhu, C. G., Sun, C. H., Xu, B., Fang, J., et al. (2009). Molecular analysis of rice plants harboring a multi-functional T-DNA tagging system. J. Genet. Genomics 36, 267-276. doi: 10.1016/S1673-8527(08)60114-9

Maruyama-Nakashita, A., Inoue, E., Watanabe-Takahashi, A., Yarnaya, T., and Takahashi, H. (2003). Transcriptome profiling of sulfur-responsive genes in gives an evidence that miR399 probably is an important regulator for multiple nutrient interactions.

\section{Acknowledgments}

This work was supported by the grants from the "Strategic Priority Research Program" of the Chinese Academy of Sciences (Grant No. XDA08010400) and Ministry of Science and Technology of China (Grant No. 2014AA10A602).

\section{Supplementary Material}

The Supplementary Material for this article can be found online at: http://www.frontiersin.org/journal/10.3389/fpls.2015. 00188/abstract
Arabidopsis reveals global effects of sulfur nutrition on multiple metabolic pathways. Plant Physiol. 132, 597-605. doi: 10.1104/pp.102.019802

Nilsson, L., Muller, R., and Nielsen, T. H. (2007). Increased expression of the MYB-related transcription factor, PHR1, leads to enhanced phosphate uptake in Arabidopsis thaliana. Plant Cell Environ. 30, 1499-1512. doi: 10.1111/j.13653040.2007.01734.x

Park, B. S., Seo, J. S., and Chua, N. H. (2014). NITROGEN LIMITATION ADAPTATION recruits PHOSPHATE2 to target the phosphate transporter PT2 for degradation during the regulation of Arabidopsis phosphate homeostasis. Plant Cell 26, 454-464. doi: 10.1105/tpc.113.120311

Raghothama, K. G. (1999). Phosphate acquisition. Ann. Rev. Plant Physiol. Plant Mol. Biol. 50, 665-693. doi: 10.1146/annurev.arplant.50.1.665

Rotte, C., and Leustek, T. (2000). Differential subcellular localization and expression of ATP sulfurylase and 5'-adenylylsulfate reductase during ontogenesis of arabidopsis leaves indicates that cytosolic and plastid forms of ATP sulfurylase may have specialized functions. Plant Physiol. 124, 715-724. doi: 10.1104/pp.124.2.715

Rubio, V., Linhares, F., Solano, R., Martin, A. C., Iglesias, J., Leyva, A., et al. (2001). A conserved MYB transcription factor involved in phosphate starvation signaling both in vascular plants and in unicellular algae. Genes Dev. 15, 2122-2133. doi: 10.1101/gad.204401

Sunkar, R., and Zhu, J. K. (2004). Novel and stress-regulated microRNAs and other small RNAs from Arabidopsis. Plant Cell 16, 2001-2019. doi: 10.1105/tpc.104.022830

Takahashi, H., Watanabe-Takahashi, A., Smith, F. W., Blake-Kalff, M., Hawkesford, M. J., and Saito, K. (2000). The roles of three functional sulphate transporters involved in uptake and translocation of sulphate in Arabidopsis thaliana. Plant J. 23, 171-182. doi: 10.1046/j.1365-313x.2000.00768.x

Takahashi, H., Yamazaki, M., Sasakura, N., Watanabe, A., Leustek, T., Engler, J. D., et al. (1997). Regulation of sulfur assimilation in higher plants: a sulfate transporter induced in sulfate-starved roots plays a central role in Arabidopsis thaliana. Pro. Natl. Acad. Sci. U.S.A. 94, 11102-11107. doi: 10.1073/pnas.94.20.11102

Xin, M., Wang, Y., Yao, Y., Xie, C., Peng, H., Ni, Z., et al. (2010). Diverse set of microRNAs are responsive to powdery mildew infection and heat stress in wheat (Triticum aestivum L.). BMC Plant Biol. 10:123. doi: 10.1186/1471-222910-123

Zhao, B., Liang, R., Ge, L., Li, W., Xiao, H., Lin, H., et al. (2007). Identification of drought-induced microRNAs in rice. Biochem. Biophys. Res. Commun. 354, 585-590. doi: 10.1016/j.bbrc.2007.01.022

Zhao, M., Ding, H., Zhu, J. K., Zhang, F., and Li, W. X. (2011). Involvement of miR169 in the nitrogen-starvation responses in Arabidopsis. New Phytol. 190, 906-915. doi: 10.1111/j.1469-8137.2011.03647.x

Zhou, J., Jiao, F. C., Wu, Z. C., Li, Y. Y., Wang, X. M., He, X. W., et al. (2008). OsPHR2 is involved in phosphate-starvation signaling and excessive phosphate accumulation in shoots of plants. Plant Physiol. 146, 1673-1686. doi: 10.1104/pp.107.111443 
Zhou, L., Liu, Y., Liu, Z., Kong, D., Duan, M., and Luo, L. (2010). Genome-wide identification and analysis of drought-responsive microRNAs in Oryza sativa. J. Exp. Bot. 61, 4157-4168. doi: 10.1093/jxb/erq237

Conflict of Interest Statement: The authors declare that the research was conducted in the absence of any commercial or financial relationships that could be construed as a potential conflict of interest.
Copyright $\odot 2015 \mathrm{Hu}$, Wang, Deng, Li, Zhang, Zhang and Chu. This is an openaccess article distributed under the terms of the Creative Commons Attribution License (CC BY). The use, distribution or reproduction in other forums is permitted, provided the original author(s) or licensor are credited and that the original publication in this journal is cited, in accordance with accepted academic practice. No use, distribution or reproduction is permitted which does not comply with these terms. 